\title{
Impact of Anesthesia on the Outcome of Acute Ischemic Stroke after Endovascular Treatment with the Solitaire Stent Retriever
}

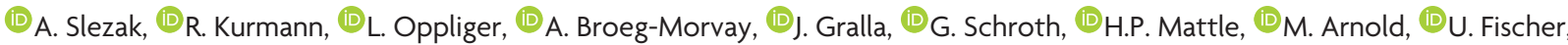

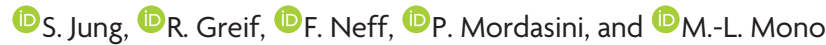

\begin{abstract}
BACKGROUND AND PURPOSE: General anesthesia during endovascular treatment of acute ischemic stroke may have an adverse effect on outcome compared with conscious sedation. The aim of this study was to examine the impact of the type of anesthesia on the outcome of patients with acute ischemic stroke treated with the Solitaire stent retriever, accounting for confounding factors.
\end{abstract}

MATERIALS AND METHODS: Four-hundred one patients with consecutive acute anterior circulation stroke treated with a Solitaire stent retriever were included in this prospective analysis. Outcome was assessed after 3 months by the modified Rankin Scale.

RESULTS: One-hundred thirty-five patients (31\%) underwent endovascular treatment with conscious sedation, and 266 patients (69\%), with general anesthesia. Patients under general anesthesia had higher NIHSS scores on admission (17 versus 13, $P<.001$ ) and more internal carotid artery occlusions ( $44.6 \%$ versus $14.8 \%, P<.001$ ) than patients under conscious sedation. Other baseline characteristics such as time from symptom onset to the start of endovascular treatment did not differ. Favorable outcome (mRS 0-2) was more frequent with conscious sedation (47.4\% versus $32 \%$; OR, $0.773 ; 95 \% \mathrm{Cl}, 0.646-0.925 ; P=.002$ ) in univariable but not multivariable logistic regression analysis $(P=.629)$. Mortality did not differ $(P=.077)$. Independent predictors of outcome were age $(\mathrm{OR}, 0.95 ; 95 \% \mathrm{Cl}, 0.933-0.969 ; P<$ $.001)$, NIHSS score (OR, $0.894 ; 95 \% \mathrm{Cl}, 0.855-0.933 ; P<.001)$, time from symptom onset to the start of endovascular treatment (OR, 0.998 ; $95 \% \mathrm{Cl}, 0.996-0.999 ; P=.011)$, diabetes mellitus (OR, 0.544; $95 \% \mathrm{Cl}, 0.305-0.927 ; P=.04)$, and symptomatic intracerebral hemorrhage (OR, $0.109 ; 95 \% \mathrm{Cl}, 0.028-0.428 ; P=.002)$.

CONCLUSIONS: In this single-center study, the anesthetic management during stent retriever thrombectomy with general anesthesia or conscious sedation had no impact on the outcome of patients with large-vessel occlusion in the anterior circulation.

ABBREVIATIONS: $\mathrm{CS}=$ conscious sedation; $\mathrm{EVT}=$ endovascular treatment; $\mathrm{GA}=$ general anesthesia

E

ndovascular treatment of acute ischemic stroke due to large-

vessel occlusion in the anterior circulation is safe and effective for improving functional outcome. ${ }^{1}$ However, there is an ongoing debate about the type of anesthesia to be used, general anesthesia (GA) or conscious sedation (CS). No patient movements, better airway control, and perceived procedural safety and efficacy are

Received December 1, 2016; accepted after revision February 14, 2017.

From the Departments of Neurology (A.S., R.K., L.O., A.B.-M., H.P.M., M.A., U.F., S.J., M.-L.M.), Diagnostic and Interventional Neuroradiology (I.G., G.S., P.M.), and Anesthesiology and Pain Medicine (R.G., F.N.), University Hospital Bern and University of Bern, Bern, Switzerland.

A. Slezak, R. Kurmann, P. Mordasini, and M.-L. Mono contributed equally to this work.

This study was supported by a grant from the Swiss Heart Foundation.

Please address correspondence to Marie-Luise Mono, MD, Department of Neurology, Inselspital, University Hospital and University of Bern, Freiburgstr, 3010 Bern,

Switzerland; e-mail: marie-luise.mono@insel.ch

http://dx.doi.org/10.3174/ajnr.A5183 regarded as potential advantages of GA, but more recent data of nonrandomized studies including 1 meta-analysis of 9 studies suggest that CS during endovascular stroke treatment might improve outcome. $^{2-5}$ This finding might be explained by a shorter time to start the intervention, less blood pressure dip, and easier neurologic monitoring during and after CS. However, many confounding factors such as stroke severity, occlusion site, pretreatment with IV rtPA, age, endovascular treatment techniques, and recanalization rates, might influence outcome. ${ }^{3,6-11}$ Recently, the results of the first randomized study, Sedation versus Intubation for Endovascular Stroke TreAtment (SIESTA), were published, which showed no differences between GA and CS for the primary end point defined as early neurologic improvement on the NIHSS after 24 hours. ${ }^{12}$

The aim of this study was to examine the impact of the type of anesthesia (GA versus CS) on the outcome of patients with acute ischemic stroke with large-vessel occlusion in the ante- 
rior circulation who were treated with the Solitaire stent retriever (Covidien, Irvine, California), while accounting for confounding factors.

\section{MATERIALS AND METHODS}

Data of 401 consecutive patients with acute ischemic stroke with large-vessel occlusion in the anterior circulation who were treated endovascularly with the Solitaire stent retriever either directly or after intravenous thrombolysis with rtPA were prospectively collected and analyzed. Patients were included between January 2010 and April 2015. The study was approved by the Cantonal Ethics Committee of Bern, and all patients or their relatives gave written consent for participation.

Endovascular treatment (EVT) was performed in 266 patients under GA and in 135 patients with CS. In 10 patients (2.5\%), CS was switched to GA. On the basis of the intention-to-treat principle, these patients were added to the CS group. Patient data (demographics, comorbidities, stroke etiology according to the Trial of Org 10172 in Acute Stroke Treatment [TOAST] criteria, ${ }^{13}$ prestroke modified Rankin Scale score, pretreatment and stroke treatment details, complications, and outcome) were recorded in the Bernese Stroke Registry.

EVT within 8 hours after symptom onset was performed immediately after CT or MR imaging under the following conditions: 1) The diagnosis of ischemic stroke was established; 2) the NIHSS score on admission assessed by a neurologist was $\geq 4$ points or isolated aphasia or hemianopsia was present or neurologic deficits recurred; 3) CT or MR angiography showed occlusion of the carotid artery, the M1 or M2 segments of the middle cerebral artery, or the A1 segment of the anterior cerebral artery; 4) hemorrhage was excluded; 5) neurologic deficits correlated with the vessel occlusion; and 6) no individual clinical or premorbid conditions or laboratory findings contraindicated EVT. When the criteria for EVT were fulfilled, digital subtraction angiography was performed via a transfemoral approach by using a biplane, high-resolution angiography system. All patients underwent complete 4-vessel cerebral angiography performed by fully trained interventional neuroradiologists. The decision for EVT in combination with intravenous thrombolysis or without it was made on an individual basis after interdisciplinary discussion. Intravenous thrombolysis was additionally given if it was indicated (symptom onset of $<4.5$ hours) and nothing against its use was indicated. The interventional neuroradiologist and neurologist decided on EVT with the Solitaire device and when necessary additional intra-arterial urokinase. At the end of angiography, recanalization was classified by the treating neuroradiologist according to the Thrombolysis in Cerebral Infarction perfusion scale grade. ${ }^{14}$

The decision of whether to perform GA or CS was made on an individual basis by the neurologist, neuroradiologist, and anesthesiologist on call considering the patient's physical status, pros and cons of the anesthetic management, and preferences of the neurointerventionalists. Reasons for choosing GA over CS were lack of cooperation or agitation of the patient, expected complexity of the interventions (eg, in case of tandem occlusions or ICA occlusions), expected difficult access anatomy, and restricted general physical or neurologic status of the patient (eg, severe stroke with need for airway protection, impaired consciousness). When a decision for CS was made, patients underwent continuous monitored anesthesia care with supplemental oxygen via a face mask and standardized monitoring including electrocardiography, pulse oximetry, end-tidal carbon dioxide measurement, and invasive arterial blood pressure measurement, which we usually measure by the side-arm extension tube of the femoral sheath to avoid loss of time for puncture of the radial artery. For sedation, intermittent IV boluses of midazolam ( $2.5 \mathrm{mg}$ ) and fentanyl (50 $\mathrm{mcg}$ ) or propofol $(20 \mathrm{mg}$ ) were given at the discretion of the attending anesthesiologist.

After proper patient positioning and preoxygenation, GA was induced with IV fentanyl (1-5 mcg/kg) and propofol (1-2 mg/ $\mathrm{kg})$, and rocuronium $(0.9 \mathrm{mg} / \mathrm{kg})$ for rapid sequence intubation. General anesthesia was maintained with IV propofol $(6-10 \mathrm{mcg} /$ $\mathrm{kg} / \mathrm{h}$ ) and boluses of fentanyl (1-3 mcg/kg) and rocuronium (0.1 $\mathrm{mg} / \mathrm{kg}$ ) according to the needs of the patient. Following tracheal intubation, the patient was mechanically ventilated until the end of the procedure (Hamilton C1; Hamilton Medical, Bonaduz, Switzerland). Patient monitoring during GA was the same as under CS. General anesthesia was terminated, and the patient was extubated as soon as possible after the intervention. All anesthesia-related data including blood pressure dips (maximum blood pressure drop within the intervention), complications during the procedure, and difficulties in patient management (eg, movement and thus conversion from sedation to general anesthesia) were recorded in the anesthetic record (AIS Release 57; COPRA System, Berlin, Germany). The goal of systolic blood pressure during EVT was between 140 and $180 \mathrm{~mm} \mathrm{Hg}$ according to our institutional standards.

After EVT, all patients were transferred to the Stroke Unit or an intermediate or intensive care unit for at least 24 hours after treatment. Neurologic status, blood pressure, glucose levels, oxygen saturation, and body temperature were monitored closely. CT or MR imaging scans were generally obtained 24 hours after treatment, and additionally in case of clinical deterioration. Symptomatic and asymptomatic intracerebral hemorrhage was graded according to the Prolyse in Acute Cerebral Thromboembolism (PROACT) II and European Cooperative Acute Stroke Study (ECASS) II criteria. ${ }^{15,16}$ Secondary preventive treatment was given according to current evidence and the American Heart Association and American Stroke Association guidelines. ${ }^{17}$ Pneumonia was diagnosed when at least 1 of the primary and 1 of the secondary criteria were fulfilled according to the US Centers for Disease Control and Prevention criteria. The primary criteria include abnormal respiratory examination and pulmonary infiltrates in chest x-rays; secondary criteria include productive cough with purulent sputum, microbiological cultures from lower respiratory tract or blood cultures, leukocytosis, and elevation of C-reactive protein. We recorded the following periprocedural complications: dissection of an extra- or intracranial vessel, perforation of an extraor intracranial vessel, and iatrogenic thrombus dislocation.

\section{Outcome}

Clinical outcome was assessed 3 months after the index event by using the modified Rankin Scale. ${ }^{18}$ The primary study end point 
Table 1: Patient characteristics and procedural details ${ }^{\mathrm{a}}$

\begin{tabular}{|c|c|c|c|}
\hline & CS & GA & $P$ Value \\
\hline No. of patients & 135 & 266 & \\
\hline Age (yr) (mean) (SD) & 70.5 (14.9) & $70.9(13.9)$ & .803 \\
\hline Age older than $80 \mathrm{yr}$ & $46(34.1)$ & $76(28.6)$ & .258 \\
\hline Male sex & 59 (43.7) & $140(52.6)$ & .091 \\
\hline Prestroke $\mathrm{mRS}<1$ & $124(94.7)$ & $228(89.8)$ & .104 \\
\hline TOAST & & & .162 \\
\hline Large-artery disease & $26(19.3)$ & $63(23.8)$ & \\
\hline Small-artery disease & $3(2.2)$ & $6(2.3)^{\prime}$ & \\
\hline Cardioembolism & 64 (47.4) & $95(35.8)$ & \\
\hline Stroke of other determined etiology & $15(11.1)$ & $23(8.7)^{\prime}$ & \\
\hline Stroke of undetermined etiology & $27(20)$ & $78(29.5)$ & \\
\hline Atrial fibrillation & $39(31.2)$ & $84(36.5)$ & .164 \\
\hline Hyperlipidemia & $59(44.4)$ & $120(45.8)$ & .786 \\
\hline Arterial hypertension & $66(48.9)$ & $146(54.9)$ & .255 \\
\hline Diabetes mellitus & $39(29.3)$ & $74(28.2)$ & .823 \\
\hline Coronary disease & $21(17.4)$ & $65(27.3)$ & .037 \\
\hline Smoking & 49 (38.9) & $111(46.3)$ & .177 \\
\hline Prior stroke or TIA & $15(14.2)$ & $32(14.4)$ & .949 \\
\hline Recurrent stroke within $3 \mathrm{mo}$ & $9(7.8)$ & $11(4.9)$ & .462 \\
\hline NIHSS on admission (mean) (SD) & $13.1(5.7)$ & $17.2(6.6)$ & $<.001$ \\
\hline Vessel occlusion site & & & $<.001$ \\
\hline ICA & $20(14.8)$ & $117(44)$ & \\
\hline $\mathrm{ACA}$ & $1(0.7)$ & $0(0)$ & \\
\hline MCA & $114(84.4)$ & $149(56)$ & \\
\hline Time from symptom onset to start of EVT (mean) (SD) (min) & $277(126)$ & 299 (157) & .165 \\
\hline Intravenous rtPA and EVT & $44(32.6)$ & $78(29.3)$ & .501 \\
\hline Mechanical thrombectomy only & $72(53.3)$ & $159(59.8)$ & .217 \\
\hline Maximal systolic blood pressure (mm Hg) (mean) (SD) & $177(25)$ & $176(25)$ & .631 \\
\hline Minimal systolic blood pressure $(\mathrm{mm} \mathrm{Hg})(\mathrm{mean})(\mathrm{SD})$ & $149(33)$ & $133(42)$ & $<.001$ \\
\hline Systolic BP gradient during procedure $(\mathrm{mm} \mathrm{Hg})($ mean) (SD) & $31(26)$ & $50(32)$ & $<.001$ \\
\hline BP-increasing drugs & $54(40.3)$ & $254(95.8)$ & $<.001$ \\
\hline BP-lowering drugs & $42(31.1)$ & $104(39.1)$ & .116 \\
\hline $\mathrm{TICI}$ 2-3 recanalization & $116(85.9)$ & $235(88.3)$ & .488 \\
\hline 3-month mRS 0-1 & $37(27.4)$ & $56(21.2)$ & .154 \\
\hline 3-month mRS 0-2 & $64(47.4)$ & $85(32)$ & .002 \\
\hline NIHSS after 24 hr (mean) (SD) & $9(8.3)^{\prime}$ & $13.9(11.2)$ & $<.001$ \\
\hline Death within 3 months & $28(20.7)$ & $77(28.9)$ & .077 \\
\hline
\end{tabular}

Note:-ACA indicates anterior cerebral artery; BP, blood pressure.

${ }^{a}$ Data are No. (\%) unless otherwise indicated.

Table 2: Peri- and postprocedural complications of endovascular treatment with the Solitaire stent retriever in anterior circulation stroke $^{\mathrm{a}}$

\begin{tabular}{lccc}
\hline & CS & GA & $P$ Value \\
\hline Pneumonia & $22(16.5)$ & $67(25.3)$ & .048 \\
Symptomatic ICH (PROACT II) & $9(6.8)$ & $20(7.6)$ & .755 \\
Symptomatic ICH (ECASS II) & $9(6.8)$ & $21(8)$ & .658 \\
Systemic bleeding & $4(3)$ & $3(1.1)$ & .185 \\
Asymptomatic ICH (PROACT II) & $24(18)$ & $52(19.9)$ & .655 \\
Peri-interventional complications & & & \\
$\quad$ Dissection & $6(4.5)$ & $12(4.6)$ & .975 \\
$\quad$ Thrombus dislocation & $12(9)$ & $33(12.5)$ & .302 \\
$\quad$ Perforation of intracranial vessels & $2(1.5)$ & $5(1.9)$ & .773 \\
\hline
\end{tabular}

Note:- $\mathrm{ICH}$ indicates intracerebral hemorrhage.

${ }^{a}$ Data are No. (\%) unless otherwise indicated.

was favorable outcome ( $\mathrm{mRS} 0-2)$. Secondary end points were excellent outcome (mRS 0-1) and death.

\section{Statistics}

Descriptive statistics are expressed as means with SDs or medians with interquartile ranges. Groups (GA and CS) were compared by the $\chi^{2}$ test for categoric variables and the Student $t$ test or, in case of a non-normal distribution, the Mann-Whitney $U$ test for continuous

\section{Outcome}

variables. Univariable logistic analysis was performed to determine an association between the type of anesthesia and outcome. To adjust for prognostic baseline factors, we performed a multivariable logistic regression including all variables with probability values of $<.1$ in univariable analysis. A probability value of $P<$ .05 was considered significant.

\section{RESULTS \\ Baseline and Treatment Characteristics}

Baseline characteristics and procedural details are given in Table 1. Coronary heart disease was more common in the GA group (27\% versus $17 \%, P=.037$ ). Patients under GA had higher NIHSS scores on admission (17 points versus 13 points, $P<.001$ ), more often had internal carotid artery occlusions $(44 \%$ versus $15 \%, P<.001$ ), received more blood pressure-elevating drugs ( $96 \%$ versus $40 \%, P<.001$ ), and experienced greater blood pressure drops during anesthesia (50 versus $31 \mathrm{~mm} \mathrm{Hg}, P<.001$ ). Patients with CS showed MCA occlusions more often $(84 \%$ versus $56 \%, P<.001)$.

Time from symptom onset to the start of EVT (277 minutes in the CS group versus 299 minutes in the GA group, $P=.165)$ and recanalization rates (TICI $2-3$ in $88 \%$ of the GA group versus $86 \%$ of the CS group, $P=.488$ ) did not differ between the groups.

\section{Peri- and Postprocedural Complications}

Peri- and postprocedural complications are summarized in Table 2. Patients under GA had pneumonia more frequently than patients under CS (25\% versus $17 \%, P=.048$ ). Other complications such as dissections, perforation of the brain-supplying arteries, or iatrogenic thrombus dislocation were equally distributed.

The distribution of the mRS scores at 3 months is presented in the Figure. Fifty-one percent of the patients with CS and $34 \%$ of patients with GA had favorable outcome (mRS 0-2) $(P=.002)$. Excellent outcome (mRS $0-1$ ) was seen in $30 \%$ of the CS and in $22 \%$ of the patients with GA $(P=.154)$. After 3 months, $22 \%$ of the patients with CS and $30 \%$ of the patients with GA had died $(P=.077)$.

In univariable analysis, GA was associated with less favorable outcome than CS (OR, 0.773; 95\% CI, 0.646-0.925; $P=.002$ ), but after adjusting for baseline factors, the difference was no longer significant (Table 3). Independent predictors of unfavorable outcome were advancing age, higher NIHSS score, longer time from symptom onset to start of EVT, diabetes mellitus, and intracerebral hemorrhage (Table 3 ). 


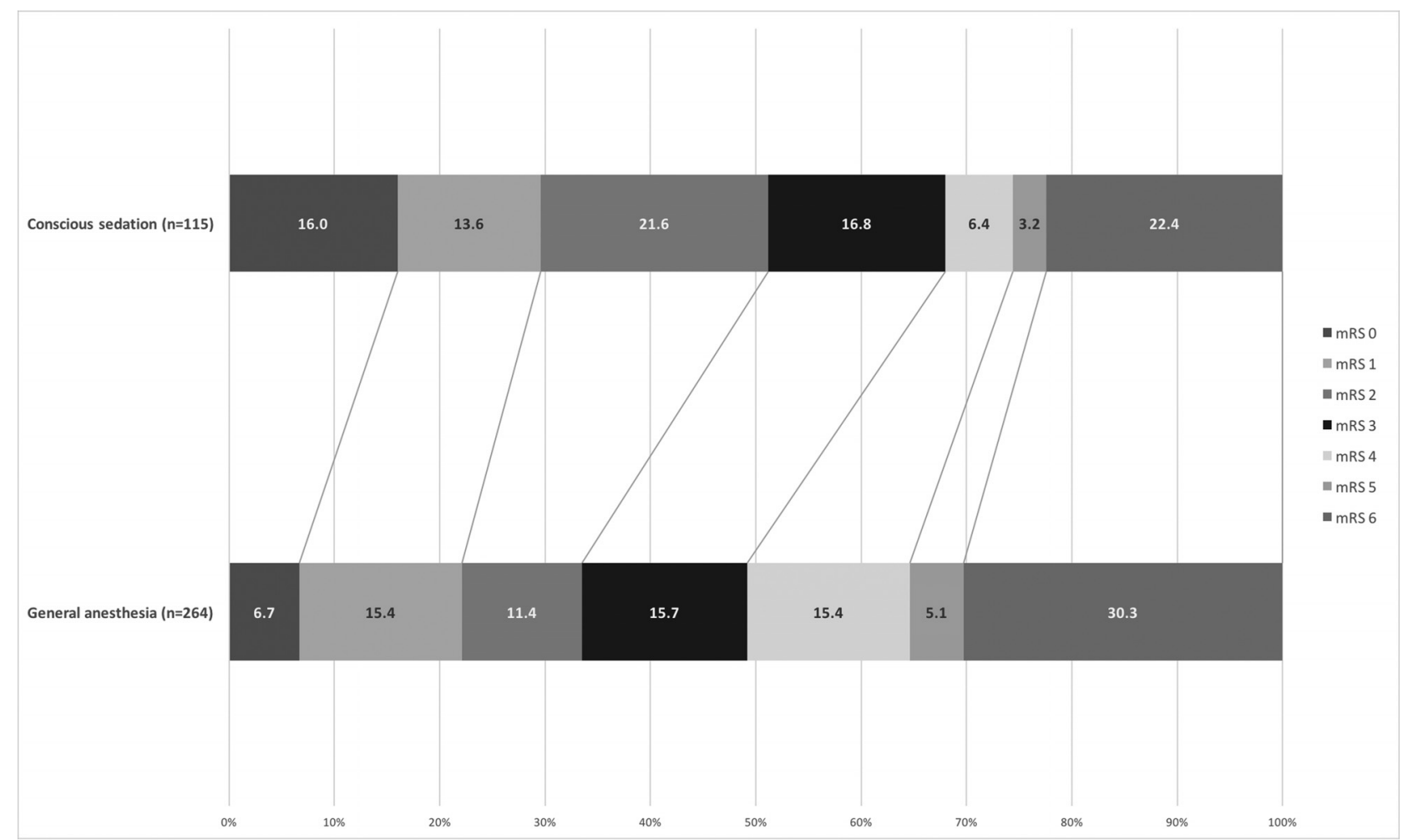

FIGURE. Three-month outcome of patients under GA compared with patients with CS. The figures in the bar indicate the percentage of patients with a given mRS at 3-month follow-up.

Table 3: Predictors of outcome at 3 months (multivariable logistic regression)

\begin{tabular}{lcc}
\hline & Odds Ratio $(95 \% \mathrm{CI})$ & $\boldsymbol{P}$ Value \\
\hline GA & $0.873(0.505-1.512)$ & .629 \\
Age & $0.95(0.932-0.969)$ & $<.001$ \\
NIHSS & $0.894(0.855-0.933)$ & $<.001$ \\
Time from symptom onset to & $0.998(0.996-0.999)$ & .01 \\
$\quad$ start of EVT & & \\
Symptomatic ICH & $0.109(0.028-0.428)$ & .002 \\
Diabetes mellitus & $0.544(0.305-0.972)$ & .04 \\
\hline
\end{tabular}

Note:- $\mathrm{ICH}$ indicates intracerebral hemorrhage.

\section{DISCUSSION}

The anesthetic management during stent retriever thrombectomy, general anesthesia, or conscious sedation has no impact on the outcome of patients with large-vessel occlusion in the anterior circulation. This is the main finding of our analysis of $>400$ consecutive patients. Univariable analysis showed an advantage for CS, but after multivariable adjustment for baseline characteristics, this difference disappeared. Also, time from symptom onset to the start of EVT, recanalization rates, and mortality did not differ between patients under CS and GA. Patients in the GA group more often had pneumonia.

Our results are in line with the findings of the SIESTA trial, a single-center randomized controlled trial. The SIESTA investigators recently reported the outcomes at 24 hours. Outcomes did not differ in patients who were treated with CS versus those under GA. Unadjusted favorable outcome after 3 months was better in patients undergoing GA than in patients treated in CS (37\% versus $18.2, P=.01)$. However, because there was no consistent shift over all mRS categories and the study was not designed to investigate long-term outcome, this result should be interpreted with caution. ${ }^{12}$ Previous studies reported that CS seems to be superior to GA for endovascular stroke treatment. ${ }^{4,5,19-22}$ A recent metaanalysis by Brinjikji et al, ${ }^{3}$ including 1956 patients from 9 nonrandomized studies, found that GA compared with CS was associated with lower odds of favorable outcome (mRS 0-2) (OR, 0.43; 95\% CI, 0.35-0.53; $P<.01$ ), lower odds of successful recanalization (OR, 0.54; 95\% CI, 0.37-0.80; $P<.01$ ), but higher odds of death (OR, 2.59; 95\% CI, 1.87-3.58, $P<.01)$ and respiratory complications (OR, 2.09; 95\% CI, 1.36-3.23; $P<.01$ ). Symptomatic and asymptomatic hemorrhage and other vascular complications were similar for both GA and CS, and time to treatment did not differ either (136 minutes for GA versus 117 minutes for CS, $P=$ .24). Baseline NIHSS scores were, on average, higher in patients with GA than for those with CS, and when the analysis was adjusted for the NIHSS score, significance got lost. ${ }^{3}$

The post hoc analysis of the Multicenter Randomized Clinical Trial of Endovascular Treatment for Acute Ischemic Stroke in the Netherlands (MR CLEAN) with balanced baseline NIHSS and occlusion sites between groups indicated higher odds of good clinical outcome with CS (unadjusted OR, 2.1; 95\% CI, 1.02$4.31 ; P=.04) .{ }^{4}$ After adjusting for prespecified prognostic factors such as age, occlusion of the internal carotid artery terminus, history of previous stroke, atrial fibrillation, diabetes mellitus, and stroke severity, the difference in outcome was lost (adjusted OR, 1.9; 95\% CI, 0.89-4.24; $P=$ not given). Furthermore, there was a significant time delay of 20 minutes with GA compared with CS $(P=.02)$. Periprocedural complications and recanalization rates did not differ between GA and CS. ${ }^{4}$

Both GA and CS have their advantages and shortcomings. One potential advantage of CS is the shorter time to treatment as 
shown in the post hoc analysis of MR CLEAN. ${ }^{4}$ In our study, the time from symptom onset to the start of EVT was slightly longer in patients undergoing GA, but this was not significant. This finding might be the result of the implementation of a standardized operating procedure to manage patients with stroke, including early notification of the anesthesiologists. These emergency procedures, involving all interprofessional and interdisciplinary health care providers, are based on the consideration of stroke as a potentially lethal and devastating threat to the health of the patient and attribute stroke management as the highest priority. Therefore, the time interval from the call for anesthesia help to needle insertion was set to 30 minutes. The possibility of monitoring the neurologic status during the procedure might be another advantage of CS. Furthermore, blood pressure remains more stable during CS. High blood pressure variability in acute stroke is associated with worse outcome. ${ }^{23}$ On the other hand, restless patients under conscious sedation may disturb the intervention, delay time to recanalization, enhance procedure-related complications, and, as a consequence, lead secondarily to GA and intubation. ${ }^{2}$ However, in our series, iatrogenic dissection, perforation of the brain-supplying arteries, or iatrogenic thrombus dislocation did not occur more often under GA.

Furthermore GA leads to more stable working conditions for the interventionalist due to reduced patient movements because of periprocedural anxiety, pain, or agitation. Therefore, the risk of procedure-related complications (eg, dissection or vessel perforation) seems to be reduced. On the other hand, GA carries the risk of drug-related arterial hypotension and cerebral blood flow reduction. $^{22}$ In our series, blood pressure drop was significantly higher under GA than with CS but had no influence on the outcome. Tracheal intubation and specifically extubation might provoke coughing or retching that raises intrathoracic and intracranial pressure, reducing cerebral blood flow and blood supply to the penumbra. Cerebral blood flow is also reduced when intubated patients are inadvertently hyperventilated leading to hypocapnia-induced vasoconstriction of the intracranial vessels. ${ }^{24}$ GA and especially an emergency switch from CS to GA carry a high risk of hypotension, aspiration, and pneumonia. As a consequence, this switch may compromise outcome in acute ischemic stroke. ${ }^{25-27}$ In our study, patients under GA had a higher risk of pneumonia, but this did not influence outcome. This result might not be the consequence of GA but rather reflect the higher morbidity of patients undergoing GA on admission. Patients undergoing GA had more severe strokes with impaired consciousness and swallowing problems baring a higher risk of aspiration already before intubation. The 10 patients in our study with conversion to GA did as well as the others.

In clinical practice, the choice of GA or CS in a given patient is an individual decision considering the patient's physical status and the complexity of the EVT. On the basis of thrombus imaging and visualization of the arteries from the aortic arch up to the pial collaterals by CTA or MRA, which are mandatory parts of our stroke protocol, patients with difficult arterial access (eg, bovine type of aortic arch, tandem occlusions, extensive thrombus burden) can be identified in advance. This identification may explain why CS had to be switched infrequently to GA (2.5\%) during the interventions in our series, avoiding time delay, complications, and, last but not least, stress for the patient and the anesthesiology and neuroradiology teams. According to the Society for Neuroscience in Anesthesiology and Critical Care Expert Consensus Statement on EVT, GA may be preferable in uncooperative or agitated patients or patients with severe strokes who need airway protection. CS seems to be feasible in cooperative patients who understand the procedure and can protect their airways and when advanced stroke imaging predicts straightforward endovascular thrombectomy. ${ }^{28}$ If GA is chosen, standardized protocols for early postprocedural neurologic assessment and early extubation should be used to minimize postextubation risks. A decisionmaking tree for selection of the anesthetic management in EVT as suggested by Dhakal et $\mathrm{al}^{29}$ may help avoid delays for EVT.

Our study has the natural limitations of selection bias in a nonrandomized single-center study. The decision as to whether to perform GA or CS was made on an individual basis, which introduces confounding factors limiting comparisons. Furthermore, reasons for the choice of the anesthetic method were not routinely documented for every patient. Therefore, underlying individual patient factors for the choice of GA or CS could not be analyzed. On the other hand, our study also has strengths, mainly the large sample size, the sole use of the Solitaire stent retriever, and strokes only in the anterior circulation. The large sample size allowed a multivariable comparison accounting for many confounding factors influencing stroke outcome.

\section{CONCLUSIONS}

Our study shows that outcome did not differ between patients treated with the Solitaire stent retriever who received either GA or CS after adjusting for baseline differences. Time from symptom onset to the start of EVT was similar for both anesthetic management choices. When we considered the pros and cons of GA and CS, the choice of the type of anesthesia seems to depend mainly on individual patient factors, such as the ability to cooperate or the need for airway protection, as well as factors facilitating the optimal conditions for the intervention team. The choice should aim for the best comfort and greatest safety of patients.

Disclosures: Agnieszka Slezak—RELATED: Grant: Swiss Heart Foundation. * Lara Oppliger-RELATED: Grant: Swiss Heart Foundation. *Jan Gralla-UNRELATED: Consulting Fee or Honorarium: Medtronic, Comments: consultant for Medtronic, global Principal Investigator of the STAR study.* Heinrich Mattle-UNRELATED: Board Membership: Steering Committee of the SWIFT PRIME study; Consultancy: Steering Committee of SWIFT PRIME. Marcel Arnold—RELATED: Grant: Swiss Heart Foundation for the Swiss Stroke Registry*; UNRELATED: Board Membership: Advisory Board for NOAK studies for: Bayer Health care, Boehringer Ingelheim, Bristol-Myers Squibb, Covidien, Daiichi Sankyo, Pfizer; Grants/Grants Pending: Swiss Heart Foundation, Swiss National Science Foundation*; Payment for Lectures Including Service on Speakers Bureaus: Bayer Health AG, Bristol-Myers Squibb, Covidien. Marie-Luise Mono-RELATED: Grant: Swiss Heart Foundation*; UNRELATED: Board Membership: Advisory Board Boehringer Ingelheim. *Money paid to the institution.

\section{REFERENCES}

1. Goyal M, Menon BK, van Zwam WH, et al; HERMES collaborators. Endovascular thrombectomy after large-vessel ischaemic stroke: a meta-analysis of individual patient data from five randomised trials. Lancet 2016;387:1723-31 CrossRef Medline

2. Brekenfeld C, Mattle HP, Schroth G. General is better than local anesthesia during endovascular procedures. Stroke 2010;41:2716-17 CrossRef Medline

3. Brinjikji W, Murad MH, Rabinstein AA, et al. Conscious sedation 
versus general anesthesia during endovascular acute ischemic stroke treatment: a systematic review and meta-analysis. AJNR Am J Neuroradiol 2015;36:525-29 CrossRef Medline

4. van den Berg LA, Koelman DL, Berkhemer OA, et al; MR CLEAN pretrial study group, Participating centers. Type of anesthesia and differences in clinical outcome after intra-arterial treatment for ischemic stroke. Stroke 2015;46:1257-62 CrossRef Medline

5. McDonald JS, Brinjikji W, Rabinstein AA, et al. Conscious sedation versus general anaesthesia during mechanical thrombectomy for stroke: a propensity score analysis. J Neurointerv Surg 2015;7: 789-94 CrossRef Medline

6. Saver JL, Jahan R, Levy EI, et al; SWIFT Trialists. Solitaire flow restoration device versus the Merci retriever in patients with acute ischaemic stroke (SWIFT): a randomised, parallel-group, non-inferiority trial. Lancet 2012;380:1241-49 CrossRef Medline

7. König IR, Ziegler A, Bluhmki E, et al; Virtual International Stroke Trials Archive (VISTA) Investigators. Predicting long-term outcome after acute ischemic stroke: a simple index works in patients from controlled clinical trials. Stroke 2008;39:1821-26 CrossRef Medline

8. Mandava P, Kent TA. A method to determine stroke trial success using multidimensional pooled control functions. Stroke 2009;40: 1803-10 CrossRef Medline

9. Smith EE, Shobha N, Dai D, et al. Risk score for in-hospital ischemic stroke mortality derived and validated within the Get With the Guidelines-stroke program. Circulation 2010;122:1496-504 CrossRef Medline

10. Linfante I, Starosciak AK, Walker GR, et al. Predictors of poor outcome despite recanalization: a multiple regression analysis of the NASA registry. J Neurointerv Surg 2016;8:224-29 CrossRef Medline

11. Zaidat OO, Fitzsimmons BF, Woodward BK, et al. Effect of a balloonexpandable intracranial stent vs medical therapy on risk of stroke in patients with symptomatic intracranial stenosis: the VISSIT randomized clinical trial. JAMA 2015;313:1240-48 CrossRef Medline

12. Schönenberger S, Uhlmann L, Hacke W, et al. Effect of conscious sedation vs general anesthesia on early neurological improvement among patients with ischemic stroke undergoing endovascular thrombectomy: a randomized clinical trial. JAMA 2016;316:1986-96 CrossRef Medline

13. Adams HP Jr, Bendixen BH, Kappelle LJ, et al. Classification of subtype of acute ischemic stroke: definitions for use in a multicenter clinical trial: TOAST-Trial of Org 10172 in Acute Stroke Treatment. Stroke 1993;24:35-41 CrossRef Medline

14. Suh SH, Cloft HJ, Fugate JE, et al. Clarifying differences among thrombolysis in cerebral infarction scale variants: is the artery half open or half closed? Stroke 2013;44:1166-68 CrossRef Medline

15. Hacke W, Kaste M, Fieschi C, et al. Randomised double-blind placebo-controlled trial of thrombolytic therapy with intravenous alteplase in acute ischaemic stroke (ECASS II): Second EuropeanAustralasian Acute Stroke Study Investigators. Lancet 1998;352: 1245-51 CrossRef Medline

16. Kase CS, Furlan AJ, Wechsler LR, et al. Cerebral hemorrhage after intra-arterial thrombolysis for ischemic stroke: the PROACT II trial. Neurology 2001;57:1603-10 CrossRef Medline
17. Powers WJ, Derdeyn CP, Biller J, et al; American Heart Association Stroke Council. 2015 American Heart Association/American Stroke Association Focused Update of the 2013 Guidelines for the Early Management of Patients with Acute Ischemic Stroke Regarding Endovascular Treatment: a Guideline for Healthcare Professionals from the American Heart Association/American Stroke Association. Stroke 2015;46:3020-35 CrossRef Medline

18. van Swieten JC, Koudstaal PJ, Visser MC, et al. Interobserver agreement for the assessment of handicap in stroke patients. Stroke 1988; 19:604-07 CrossRef Medline

19. Abou-Chebl A, Lin R, Hussain MS, et al. Conscious sedation versus general anesthesia during endovascular therapy for acute anterior circulation stroke: preliminary results from a retrospective, multicenter study. Stroke 2010;41:1175-79 CrossRef Medline

20. Davis MJ, Menon BK, Baghirzada LB, et al; Calgary Stroke Program. Anesthetic management and outcome in patients during endovascular therapy for acute stroke. Anesthesiology 2012;116:396-405 CrossRef Medline

21. Jumaa MA, Zhang F, Ruiz-Ares G, et al. Comparison of safety and clinical and radiographic outcomes in endovascular acute stroke therapy for proximal middle cerebral artery occlusion with intubation and general anesthesia versus the nonintubated state. Stroke 2010;41:1180-84 CrossRef Medline

22. Li F, Deshaies EM, Singla A, et al. Impact of anesthesia on mortality during endovascular clot removal for acute ischemic stroke. J Neurosurg Anesthesiol 2014;26:286-90 CrossRef Medline

23. Stead LG, Gilmore RM, Vedula KC, et al. Impact of acute blood pressure variability on ischemic stroke outcome. Neurology 2006; 66:1878-81 CrossRef Medline

24. Takahashi C, Liang CW, Liebeskind DS, et al. To tube or not to tube? The role of intubation during stroke thrombectomy. Front Neurol 2014;5:170 CrossRef Medline

25. Hassan AE, Chaudhry SA, Zacharatos $\mathrm{H}$, et al. Increased rate of aspiration pneumonia and poor discharge outcome among acute ischemic stroke patients following intubation for endovascular treatment. Neurocrit Care 2012;16:246-50 CrossRef Medline

26. Koennecke HC, Belz W, Berfelde D, et al; Berlin Stroke Register Investigators. Factors influencing in-hospital mortality and morbidity in patients treated on a stroke unit. Neurology 2011;77:965-72 CrossRef Medline

27. Li J, Murphy-Lavoie H, Bugas C, et al. Complications of emergency intubation with and without paralysis. Am J Emerg Med 1999;17: 141-43 CrossRef Medline

28. Talke PO, Sharma D, Heyer EJ, et al. Republished: Society for Neuroscience in Anesthesiology and Critical Care expert consensus statement-a management of endovascular treatment for acute ischemic stroke. Stroke 2014;45:e138-150 CrossRef Medline

29. Dhakal LP, Díaz-Gómez JL, Freeman WD. Role of anesthesia for endovascular treatment of ischemic stroke: do we need neurophysiological monitoring? Stroke 2015;46:1748-54 CrossRef Medline 